\title{
On the Optimal Design of Linear Networked Systems
}

\author{
V. Azhmyakov ${ }^{*, \dagger}$ R. Galvan-Guerra* R. Velazquez Cuevas* \\ A.S. Poznyak * \\ * Departamento de Control Automatico, CINVESTAV, A.P. 14-740, \\ CP 07000, Mexico D.F., Mexico (Tel: +52 55-5747-3740; \\ e-mail:vazhmyakov@ctrl.cinvestav.mx) \\ $\dagger$ Systems and Control Theory Group, Max Planck Institute for \\ Dynamics of Complex Technical Systems, Sandtorstr. 1, \\ D-39106 Magdeburg, Germany
}

\begin{abstract}
This paper addresses the problem of optimal design for linear networked control systems (NCSs). We deal with the delayed NCSs in the continuous time-domain and propose a computational approach to optimization problems based on the hybrid LQ-type techniques. In particular, we develop an explicit connection between the networked control processes and the corresponding hybrid systems. For the constructive feedback control design procedure we derive the necessary Riccati-formalism and propose an implementable solution algorithm.
\end{abstract}

Keywords: networked systems, hybrid systems, LQ optimization

\section{INTRODUCTION}

Conventional control systems are composed of interconnected controllers, sensors and actuators following a pointto-point architecture. As an alternative to this architecture we could use a networked or distributed architecture that offer a more efficient reconfigurability, resource utilization and reduce the installation and maintenance cost. The systems that follow this architecture are named networked control systems (see e.g., [11]). This paper deals with the problem of optimal design for a class of NCSs where the control loops are closed through a real-time network, forming a delay control system. Since an implementable control system possesses some stability properties, we also incorporate into the above optimal control design procedure an auxiliary stability analysis. It is well-known that the ability to operate a delayed control system in an optimal way remains a challenging theoretical task. Indeed, the time delays are common in mathematical models of industrial processes, and the theory of continuous time systems with time delay is complicated (see e.g., [1, 21]). In this context and with focus on particular classes, many schemes have been proposed to tackle and analyze the problem. For a deeper discussion on the main theoretical and computational results see e.g., $[11,17,26,8]$.

In the last years, there has been a revival of the first-order optimization techniques and related numerical schemes based on the corresponding hybrid version of the Maximum Principle (MP) see e.g., [2, 5]. For a classical optimal control problem (OCP) governed by a closed-loop control system, one of the main tools toward the construction of optimal trajectories is the celebrated Bellman Dynamic Programming (DP) method (see e.g., [9, 10]). It is wellknown that for a conventional OCP the DP approach is equivalent to the techniques based on the usual Pon- tryagin MP(see e.g., [13, 20]). This fact is due to their numerical robustness, reliability and the existence of well established convergence results. The aim of this contribution is to develop an optimal control law that stabilize an unstable system using a hybrid control technique and the corresponding Riccati-formalism [6] (similarly to the conventional LQ-theory $[13,20])$. We also discuss some advanced numerical schemes based on the above hybrid Riccati approach.

The remainder of this paper is organized as follows. In the next section we give a short introduction to a class of the NCSs under consideration. Section 3 is devoted to the newly elaborated Riccati-based techniques and concepts for the hybrid OCPs. Our aim is to apply the hybrid version of the LQ approach to optimal design of linear NCSs. Section 4 contains a constructive solution procedure for OCPs which determined an approximate optimal behavior of NCSs. Finally, we discuss the computational aspect of the proposed methodology for optimization of networked systems, establish the stability properties of the generated closed-loop system and study an illustrative example. Section 5 summarize the paper.

\section{NETWORKED CONTROL SYSTEMS: BASIC CONCEPTS}

A NCS architecture consists of numerous physical and computational elements or "agents", which have physical and informational interactions and dependencies, supported by multiple, overlapping network resources. Let us consider the following analytical concept proposed in [11].

Definition 1. A NCS architecture is a three tuple

$$
N C S=\{D(\tau), I, T(p)\},
$$

where 
- $D(\tau)$ : agent dynamics with possible time-delays $\tau$ which represent a collection of linear hybrid systems that can be impacted

a) directly by continuous and discrete states/outputs from other agents,

b) indirectly by the receipt of state or output information from other agents over one or more communication networks,

c) by internal and external elements and (possible) disturbances.

- I: network information flows, represented by a directed graph. This graph summarized the information dependencies detailed in the dynamics equations.

- $T(p)$ : network topology, represented by a colored, directed p-nodes graph. This graph summarizes the communication resources in terms of the suitable links/networks.

The presented definition can be considered, for example, as a suitable conceptual model for the architecture of the simple delayed networked control system with the "initial" and "causal" delays $\tau_{a}$ and $\tau$ from $D(\tau)$ (see Fig. 1).

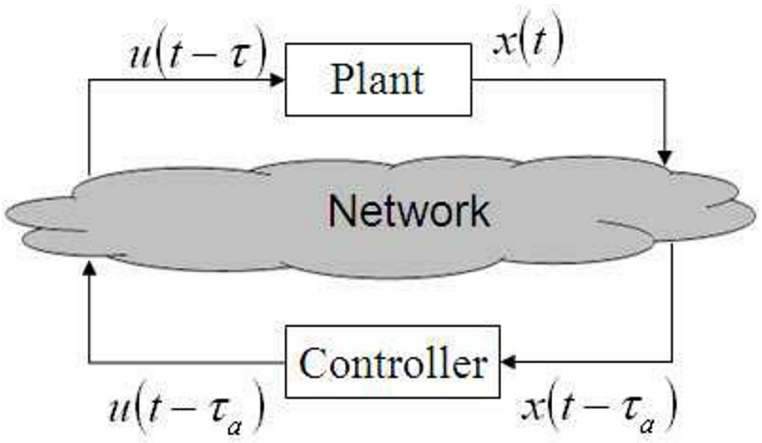

Fig. 1. A simple NCS with delays

Let consider a classical (non-networked) linear system $\dot{x}(t)=A x(t)$. It is well-known that the global stability analysis of this linear system is closely related to existence of a positive defined matrix $P$ such that $A^{T} P+P A=-Q$ for a given positive defined matrix $Q$. A basic tool for the the stability analysis of the delayed linear systems is given by the analogous result presented in [11]. The maximal bound for the time delay $\tau$ from Definition 1 can be estimated with help of the corresponding Lyapunov function for the (non-networked) auxiliary linear dynamical system and is equal to

$$
\frac{\lambda_{\min }(Q)}{16 \lambda_{2} \sqrt{\left(\frac{\lambda_{2}}{\lambda_{1}}\left\|A_{N C S}\right\|^{2} \sqrt{\left(\frac{\lambda_{2}}{\lambda_{1}}+1\right)} \sum_{i=1}^{p} i\right.}},
$$

where $A_{N C S}$ is a system matrix of the networked system, $\lambda_{1}$ and $\lambda_{2}$ are the minimal and maximal eigenvalues of $P$ and $p$ is the number of the nodes (see Definition 1). Under the above upper bound for $\tau$ one can guarantee the exponential stability of the NCS under consideration. Finally, we also refer to [18] for an alternative approach based on the "switched" Lyapunov functions for some alternative classes of hybrid systems.

\section{OPTIMIZATION OF HYBRID SYSTEMS}

Let us start by introducing a variant of the general concept for linear hybrid systems with autonomous location transitions from $[4,7]$.

Definition 2. A linear hybrid system is a 7-tuple $\{\mathcal{Q}, \mathcal{X}, U, A, B, \mathcal{U}, \mathcal{S}\}$

where

- $\mathcal{Q}$ is a finite set of discrete states (called locations);

- $\mathcal{X}=\left\{\mathcal{X}_{q}\right\}, q \in \mathcal{Q}$, is a family of state spaces such that $\mathcal{X}_{q} \subseteq \mathbb{R}^{n}$

- $U \subseteq \mathbb{R}^{m}$ is a set of admissible control input values (called control set);

- $A=\left\{A_{q}(\cdot)\right\}, B=\left\{B_{q}(\cdot)\right\}, q \in \mathcal{Q}$ are families of continuously differentiable matrix-functions

$$
A_{q}: \mathbb{R} \rightarrow \mathbb{R}^{n \times n}, B_{q}: \mathbb{R} \rightarrow \mathbb{R}^{n \times m}
$$

- $\mathcal{U}$ is the set of all admissible control functions;

- $\mathcal{S}$ is a subset of $\Xi$, where

$$
\Xi:=\left\{\left(q, x, q^{\prime}, x^{\prime}\right): q, q^{\prime} \in \mathcal{Q}, x \in \mathcal{X}_{q}, x^{\prime} \in \mathcal{X}_{q^{\prime}}\right\} .
$$

A linear hybrid system from Definition 2 is defined on a finite time-interval $\left[0, t_{f}\right]$. We refer to $[22,23,24,25]$ for some abstract concepts of hybrid systems. Let $U$ be a convex and closed set. We also assume that

$$
\mathcal{U}:=\left\{u(\cdot) \in \mathbb{L}_{m}^{\infty}\left(0, t_{f}\right) \mid u(t) \in U \text { a.e. on }\left[0, t_{f}\right]\right\},
$$

where $\mathbb{L}_{m}^{\infty}\left(0, t_{f}\right)$ is the standard Lebesgue space of measurable and essentially bounded functions. In this paper, we suppose that some affine functions

$$
m_{q, q^{\prime}}: \mathbb{R}^{n} \rightarrow \mathbb{R}, q, q^{\prime} \in \mathcal{Q}, m_{q, q^{\prime}}(x)=b_{q, q^{\prime}} x+c_{q, q^{\prime}}
$$
are given such that the hyperplanes

$$
M_{q, q^{\prime}}:=\left\{x \in \mathbb{R}^{n}: m_{q, q^{\prime}}(x)=0\right\}
$$

are pairwise disjoint. Here $b_{q, q^{\prime}} \in \mathbb{R}^{n}$ and $c_{q, q^{\prime}} \in \mathbb{R}$ for every $q, q \in \mathcal{Q}$. The given hyperplanes $M_{q, q^{\prime}}$ represents the (affine) switching sets at which a switch from location $q$ to location $q^{\prime}$ can take place. We say that a location switching from $q$ to $q^{\prime}$ occurs at a "switching time". We now consider a linear hybrid system with $r \in \mathbb{N}$ switching times $\left\{t_{i}\right\}, i=1, \ldots, r$, where

$$
0=t_{0}<t_{1}<\ldots<t_{r-1}<t_{r}=t_{f} .
$$

Note that the above sequence of switching times $\left\{t_{i}\right\}$ is not defined a priory. A hybrid control system remains in location $q_{i} \in \mathcal{Q}$ for all $t \in\left[t_{i-1}, t_{i}[, i=1, \ldots, r\right.$. In the following, we recall the notion of hybrid trajectory of the systems under consideration (see e.g., [4]).

Definition 3. An admissible hybrid trajectory associated with a given linear hybrid system from Definition 2 is a triple $\mathbf{X}=\left(x(\cdot),\left\{q_{i}\right\}, \tau\right)$, where $x(\cdot)$ is a continuous part of trajectory, $\left\{q_{i}\right\}_{i=1, \ldots, r}$ is a finite sequence of locations and $\tau$ is the corresponding sequence of switching times such that $x(0)=x_{0} \notin \bigcup_{q \in \mathcal{Q}} M_{q}$ and for each $i=1, \ldots, r$ and every admissible control $u(\cdot) \in \mathcal{U}$ we have

- $x_{i}(\cdot)=\left.x(\cdot)\right|_{\left(t_{i-1}, t_{i}\right)}$ is an absolutely continuous function on $\left(t_{i-1}, t_{i}\right)$ continuously prolongable to closed intervals $\left[t_{i-1}, t_{i}\right], i=1, \ldots, r$;

- $\dot{x}_{i}(t)=A_{q_{i}}(t) x_{i}(t)+B_{q_{i}}(t) u_{i}(t)$ for almost all time instants $t \in\left[t_{i-1}, t_{i}\right]$, where $u_{i}(\cdot)$ is a restriction of the chosen control function $u(\cdot)$ on the time interval $\left[t_{i-1}, t_{i}\right]$. 
A linear hybrid system in the sense of Definitions 2 and Definition 3 that satisfies all above assumptions is denoted by $\mathcal{L} H S$. Note that the pair $(q, x(t))$ represents the hybrid state at time $t$, where $q$ is a location $q \in \mathcal{Q}$ and $x(t) \in \mathbb{R}^{n}$. Definition 3 describes the dynamic of a hybrid control system $\mathcal{L} H S$. Since $x(\cdot)$ is a continuous function, Definition 3 describes a class of hybrid systems without impulse components of the (continuous) trajectories. Therefore, the corresponding switching sets $M_{q, q^{\prime}}$ are defined for

$$
x\left(t_{i}\right)=x\left(t_{i+1}\right), i=1, \ldots, r-1 .
$$

Under the above assumptions, for each admissible control $u(\cdot) \in \mathcal{U}$ and for every interval $\left[t_{i-1}, t_{i}\right]$ (for every location $\left.q_{i} \in \mathcal{Q}\right)$ there exists a unique absolutely continuous solution of the linear differential equations from Definition 3 . This means that for each $u(\cdot) \in \mathcal{U}$ we have a unique absolute continuous trajectory of $\mathcal{L} H S$. Moreover, the switching times $\left\{t_{i}\right\}$ and the discrete trajectory $\left\{q_{i}\right\}$ for a hybrid control system $\mathcal{L} H S$ are also uniquely defined. Note that the evolution equation for the trajectory $x(\cdot)$ of a given linear hybrid system $\mathcal{L} H S$ can also be represented as follows

$$
\begin{aligned}
& \dot{x}(t)=\sum_{i=1}^{r} \beta_{\left[t_{i-1}, t_{i}\right)}(t)\left(A_{q_{i}}(t) x_{i}(t)+B_{q_{i}}(t) u_{i}(t)\right) \\
& \text { a.e. on }\left[0, t_{f}\right], x(0)=x_{0}
\end{aligned}
$$

where $\beta_{\left[t_{i-1}, t_{i}\right)}(\cdot)$ is the characteristic function of the interval $\left[t_{i-1}, t_{i}\right)$ for $i=1, \ldots, r$. Let $S_{f}: \mathbb{R} \rightarrow \mathbb{R}^{n \times n}$, $S_{q}: \mathbb{R} \rightarrow \mathbb{R}^{n \times n}$ and $R_{q}: \mathbb{R} \rightarrow \mathbb{R}^{m \times m}$, where $q \in \mathcal{Q}$. Assume that $S_{f}$ is symmetric and positive semidefinite, and that for every $t \in\left[0, t_{f}\right]$ and every $q \in \mathcal{Q}$ the matrix $S_{q}(t)$ is also a symmetric and positive semidefinite matrix. Moreover, let $R_{q}(t)$ be a symmetric and positive definite for every $t \in\left[0, t_{f}\right]$ and every $q \in \mathcal{Q}$. We also assume that the given matrix-functions $S_{q}(\cdot), R_{q}(\cdot)$ are continuously differentiable. Given a system $\mathcal{L} H S$ we consider the following hybrid linear quadratic (HLQ) problem:

$$
\begin{aligned}
& \operatorname{minimize} J(u(\cdot), x(\cdot)):=\frac{1}{2}\left(x_{r}^{T}\left(t_{f}\right) S_{f} x_{r}\left(t_{f}\right)\right)+ \\
& \frac{1}{2} \sum_{i=1}^{r} \int_{t_{i-1}}^{t_{i}}\left(x_{i}^{T}(t) S_{q_{i}}(t) x_{i}(t)+u_{i}^{T}(t) R_{q_{i}}(t) u_{i}(t)\right) d t
\end{aligned}
$$

over all admissible trajectories $\mathbf{X}$ of $\mathcal{L} H S$.

Evidently, (1) is the problem of minimizing the quadratic Bolza cost functional $J$ over all trajectories of the given linear hybrid system. Note that we study the hybrid OCP (1) in the absence of possible target and state constraints. Throughout the paper we assume that the HLQ problem (2) has an optimal solution $\left(u^{\text {opt }}(\cdot), \mathbf{X}^{\text {opt }}(\cdot)\right)$, where $u^{o p t}(\cdot) \in \mathcal{U}$ and $\mathbf{X}^{o p t}(\cdot)$ belongs to the set of admissible trajectories from Definition 3. It is necessary to stress that the existence of an optimal pair $\left(u^{\text {opt }}(\cdot), \mathbf{X}^{\text {opt }}(\cdot)\right)$ for a HLQ problem of the above type follows from the general existence theory for linear quadratic OCPs with a convex closed control set $U$ (see e.g., $[20]$ ). We now apply the HMP (see [4]) to the HLQ problem under consideration and formulate the corresponding necessary optimality conditions. For general optimality conditions in the form of a HMP see also $[4,14,23,24,25]$.

Theorem 1. Let $\left(u^{\text {opt }}(\cdot), \mathbf{X}^{\text {opt }}(\cdot)\right)$ be an optimal solution of the regular OCP $(2)$. Then there exist absolutely continuous functions $\psi_{i}(\cdot)$ on the time intervals $\left(t_{i-1}^{o p t}, t_{i}^{o p t}\right)$, where $i=1, \ldots, r$, and a nonzero vector of Lagrange multipliers $a=\left(a_{1}, \ldots, a_{r-1}\right)^{T} \in \mathbb{R}^{r-1}$ such that

$$
\begin{aligned}
& \dot{\psi}_{i}(t)=-A_{q_{i}}^{T}(t) \psi_{i}(t)+S_{q_{i}}(t) x_{i}^{o p t}(t) \\
& \text { a. e. on }\left[t_{i-1}^{o p t}, t_{i}^{o p t}\right] \\
& \psi_{r}\left(t_{f}\right)=-S_{f} x_{r}^{o p t}\left(t_{f}\right),
\end{aligned}
$$

and

$$
\begin{aligned}
& \psi_{i}\left(t_{i}^{o p t}\right)=\psi_{i+1}\left(t_{i}^{o p t}\right)+a_{i} \frac{d m_{q_{i}, q_{i+1}}\left(x_{i}^{o p t}\left(t_{i}^{o p t}\right)\right)}{d x_{i}} \\
& =\psi_{i+1}\left(t_{i}^{o p t}\right)+a_{i} b_{q_{i}, q_{i+1}}
\end{aligned}
$$

where $i=1, \ldots, r-1$. Moreover, for every admissible control $u(\cdot) \in \mathcal{U}$ the partial Hamiltonian

$$
\begin{aligned}
& H_{q_{i}}(t, x, u, \psi):=\left\langle\psi_{i}, A_{q_{i}}(t) x_{i}+B_{q_{i}}(t) u_{i}\right\rangle- \\
& \frac{1}{2}\left(x_{i}^{T} S_{q_{i}}(t) x_{i}+u_{i}^{T} R_{q_{i}}(t) u_{i}\right) .
\end{aligned}
$$

satisfies the following maximality conditions

$$
\begin{aligned}
& \max _{u \in U} H_{q_{i}}\left(t, x^{o p t}(t), u, \psi(t)\right)= \\
& H_{q_{i}}\left(t, x^{o p t}(t), u^{o p t}(t), \psi(t)\right), \quad t \in\left[t_{i-1}^{o p t}, t_{i}^{o p t}\right),
\end{aligned}
$$

where $i=1, \ldots, r$ and $\psi(t):=\sum_{i=1}^{r} \beta_{\left[t_{i-1}^{o p t}, t_{i}^{o p t}\right)}(t) \psi_{i}(t)$ for all $t \in\left[0, t_{f}\right]$.

Note that the adjoint variable $\psi(\cdot)$ is an absolutely continuous function on every open time intervals $\left(t_{i-1}^{\text {opt }}, t_{i}^{\text {opt }}\right)$ for $i=1, \ldots, r$ but discontinuous at the switching points $t_{i}^{\text {opt }} \in \tau^{\text {opt }}$. On the other hand, we are able to establish the continuity properties of the "full" optimal Hamiltonian

$$
\tilde{H}^{o p t}(t):=\sum_{i=1}^{r} \beta_{\left[t_{i-1}^{o p t}, t_{i}^{o p t}\right)}(t) H_{q_{i}}\left(t, x^{o p t}(t), u^{o p t}(t), \psi(t)\right)
$$

computed for optimal pair $\left(u^{o p t}(\cdot), \mathbf{X}^{o p t}(\cdot)\right)$ and for the corresponding adjoint variable $\psi(\cdot)$.

Theorem 2. Under assumptions of Theorem 1, the "full" optimal Hamiltonian $\tilde{H}^{o p t}(\cdot)$ introduced above is a continuous function of time.

The proof of the presented theorem can be found in [6]. We also refer to [24] for a similar "continuity" result established for some other classes of hybrid systems. Let us now consider the linear boundary value problem (1)(3) for $U \equiv \mathbb{R}^{m}$. The maximization condition (5) from the above HMP (Theorem 1) implies that

$$
u_{i}^{o p t}(t)=R_{q_{i}}^{-1}(t) B_{q_{i}}^{T}(t) \psi_{i}(t), t \in\left[t_{i-1}^{o p t}, t_{i}^{o p t}\right) .
$$

Using this representation of an optimal control and the basic facts from the theory of linear differential equations, we now compute (similarly to $[13,20]$ ) an optimal control $u^{o p t}(\cdot)$ for (2) in the form of an optimal partially linear feedback control law

$$
\begin{aligned}
& u^{o p t}(t)=-C(t) x^{o p t}(t)= \\
& -\sum_{i=1}^{r} \beta_{\left[t_{i-1}, t_{i}\right)}(t) C_{i}(t) x_{i}^{o p t}(t),
\end{aligned}
$$

where $C_{i}(t):=R_{q_{i}}^{-1}(t) B_{q_{i}}^{T}(t) P_{i}(t)$ is a partial gain matrix and $P_{i}(\cdot)$ is the partial Riccati matrix associated with every location $q_{i}^{\text {opt }} \in \mathcal{Q}$. Analogously to the classic case, for every location $q_{i}^{\text {opt }} \in \mathcal{Q}$ and for almost all $t \in\left(t_{i-1}^{\text {opt }}, t_{i}^{\text {opt }}\right)$ we obtain the differential equation 


$$
\begin{aligned}
& \dot{P}_{i}(t)+P_{i}(t) A_{q_{i}}(t)+A_{q_{i}}^{T}(t) P_{i}(t)- \\
& P_{i}(t) B_{q_{i}}(t) R_{q_{i}}^{-1}(t) B_{q_{i}}^{T}(t) P_{i}(t)+S_{q_{i}}(t)=0,
\end{aligned}
$$

known as the Riccati matrix differential equation. We call this equation the partial Riccati equation. Evidently, every matrix $C_{i}(\cdot)$ and every matrix $P_{i}(\cdot)$ and the corresponding partial Riccati equation (7) are associated with a current location $q_{i} \in \mathcal{Q}$ of the given $\mathcal{L} H S$. We also can deduce the usual relations

$$
\psi_{i}(t)=-P_{i}(t) x_{i}^{o p t}(t)
$$

for $t \in\left[t_{i-1}^{o p t}, t_{i}^{o p t}\right.$ ) and $i=1, \ldots, r$. A symmetric (for all variables $t \in\left[0, t_{f}\right]$ ) hybrid Riccati matrix

$$
P(t):=\sum_{i=1}^{r} \beta_{\left[t_{i-1}^{o p t}, t_{i}^{o p t}\right)}(t) P_{i}(t)
$$

which satisfies all equations (7) and the boundary (terminal) condition $P\left(t_{f}\right)=S_{f}$, gives rise to the optimal feedback dynamics of (1) determined by the above partially linear feedback control function (6). The investigation of the family of equations $(7)$ on the full time interval $\left[0, t_{f}\right]$ also involves the "continuity" question associated with the above-introduced hybrid Riccati matrix $P(\cdot)$. Evidently, the continuity/smoothness of a value function is a question of general interest also in the context of other classes of OCPs governed by linear or nonlinear hybrid systems. Related to the above presented optimization theory for a $\mathcal{L} H S$ we are now able to formulate our main theoretical result, namely, the discontinuity of the hybrid Riccati matrix $P(\cdot)$.

Theorem 3. Under assumptions of Theorem 1, the hybrid Riccati matrix $P(\cdot)$ is a discontinuous function on $\left[0, t_{f}\right]$.

Proof: Assume that $P(\cdot)$ is continuous on the time interval $\left[0, t_{f}\right]$. In particular, this means that

$$
P_{i}\left(t_{i}^{o p t}\right)=P_{i+1}\left(t_{i}^{o p t}\right) \quad \forall i=1, \ldots, r-1 .
$$

Using the above continuity assumption for $P(\cdot)$, the continuity of $x(\cdot)$ and the formula for the adjoint variable, we deduce that $\psi_{i}\left(t_{i}^{o p t}\right)=-\lim _{t \uparrow t_{i}^{o p t}} P_{i}(t) x_{i}^{o p t}(t)$ and $\psi_{i+1}\left(t_{i}^{o p t}\right)=-\lim _{t \downarrow t_{i}^{o p t}} P_{i+1}(t) x_{i+1}^{o p t}(t)$. Then from the relation $\psi_{i}(t)=-P_{i}(t) x_{i}^{o p t}(t)$ and from the jump conditions (4) for the adjoint variables $\psi(\cdot)$ (Theorem 1) we obtain: $-P_{i}\left(t_{i}^{o p t}\right) x^{o p t}\left(t_{i}^{o p t}\right)=-P_{i+1}\left(t_{i}^{o p t}\right) x^{o p t}\left(t_{i}^{o p t}\right)+a_{i} b_{q_{i}, q_{i+1}}$, where $i=1, \ldots, r-1$.. Hence

$$
\left[P_{i+1}\left(t_{i}^{o p t}\right)-P_{i}\left(t_{i}^{o p t}\right)\right] x_{i}\left(t_{i}^{o p t}\right)=a_{i} b_{q_{i}, q_{i+1}} .
$$

Since $x^{o p t}(\cdot)$ is continuous and the obtained vector of optimal Lagrange multipliers $a=\left(a_{1}, \ldots, a_{r-1}\right)^{T}$ is nontrivial, the function $P(\cdot)$ is a discontinuous function on $\left[0, t_{f}\right]$. The obtained contradiction completes the proof.

It is necessary to stress that the partial Riccati equation (7) can also be derived using the general Bellman approach for hybrid systems (see [15]).

\section{OPTIMAL DESIGN OF THE LINEAR NCSS}

Consider the following model of a linear NCS

$$
\begin{aligned}
& \dot{x}(t)=A x(t)+B u(t), \quad t>0, \\
& x(0)=x_{0},
\end{aligned}
$$

where $x(t) \in \mathbb{R}^{n}$ is the state vector and $u(t) \in \mathbb{R}^{m}$ is the control vector. Let $\tau_{k} \in D(\tau), k=0,1, \ldots$ be possible delays (in the sense of Definition 1). Motivated by the optimal partially linear feedback control strategies from Section 4, we now choose the admissible control strategy in the form

$$
u_{k}(t)=-K(t) x\left(t-\tau_{k}\right), \quad t \geq \tau_{0}
$$

Note that for $0 \leq t<\tau_{0}$ the control low $u_{0}(\cdot)$ is assumed to be prescribed. This make it possible to represent (9) as a closed-loop system

$$
\begin{aligned}
& \dot{x}(t)=A x(t)-B K(t) \hat{x}_{k}(t), \quad t>0, \\
& \hat{x}_{k}(t):=x\left(t-\tau_{k}\right) .
\end{aligned}
$$

For the newly introduced "state" $\hat{x}_{k}(\cdot)$ we have the following relation

$$
\hat{x}_{k}(t)=e^{-A \tau_{k}} x(t)+\int_{0}^{\tau_{k}} e^{-s A} B K(t+s) \hat{x}_{k}(t+s) d s
$$

where $\tau_{k} \in D(\tau)$. Substituting (11) in (9) we now obtain

$$
\begin{aligned}
& \dot{x}(t)=\left(A-B K(t) e^{-A \tau_{k}}\right) x(t)- \\
& B K(t) \int_{0}^{\tau_{k}} e^{-s A} B K(t+s) \hat{x}_{k}(t+s) d s .
\end{aligned}
$$

The resulting dynamical system (12) can now be interpreted as a linear hybrid system with controlled switchings (see [24] for details)

$$
\dot{x}(t)=A_{k} x(t)+g_{k}(t) \quad t \in\left[t_{k-1}, t_{k}\right)
$$

where $A_{k}:=A-B K e^{-A \tau_{k}}$, and

$$
g_{k}(t):=B K(t) \int_{0}^{\tau_{k}} e^{-s A} B K(t+s) \hat{x}_{k}(t+s) d s .
$$

The time sequence $\left\{t_{k}\right\}, t_{k} \in \mathbb{R}_{+}$for $k=0,1, \ldots$ indicates here the intervals of constant system-delays (for example, on the time interval $\left[t_{k-1}, t_{k}\right)$ the closed-loop system (12) is a $\tau_{k}$-delayed system).

We now consider a HLQ problem of the type (2) for the obtained linear hybrid system (13) with some constant matrices $R_{k}, S_{k}$ Note that system (13) is written in a closedloop form and the problem is to select the gain matrix $K(\cdot)$ in an optimal manner. Using the developed LQ-type theory for hybrid systems optimization (see Section 3), we now choose a quasi-optimal gain matrix $K(\cdot)$ in the following form $K(t)=R_{k}^{-1}(t) B^{T}(t) P_{k}(t)$, where $P_{k}(\cdot)$ is a solution of the Riccati equations of the type (7), and examine the resulting system (13). Evidently, the proposed selection of the matrix $K(\cdot)$ corresponds to a quasi-optimal feedback control in the sense of the HLQ problem stated for system (13). The corresponding behavior of system (13) approximates a LQ-optimal dynamics of the initial timedelated NCS (9) in the case of "small" delays $\tau_{k}$ from $D(\tau)$. Note that similarly to the conventional LQ optimization theory the stability properties of a LQ-optimal linear hybrid system is a consequence of the optimality condition (see [] for theoretical details). In the case of the obtained quasi-optimal strategy for $K(\cdot)$ we now need to guarantee the stability of (13) by a special analytical result. Usually stability results for hybrid and switched systems are natural extensions of the well-known Lyapunov techniques. Let us present a Lyapunov-based stability criterion from [12] which can be applied to the corresponding analysis of the resulting system (13) with the chosen matrix $K(\cdot)$.

Theorem 4. Suppose we have candidate Lyapunov functions $V_{k}\left(V_{k}\right.$ are positive definite and $\dot{V}_{k}(x(t)) \leq 0$ for all $\left.t \in\left[t_{k-1}, t_{k}\right)\right)$. Let $\mathcal{S}$ be a set of all switching sequences 
associated with the system. If for each element from $\mathcal{S}$ we have that for all $k=0,1, \ldots, V_{k}$ is Lyapunov-like for $A_{k}$ and $g_{k}$, then the system is stable.

We also refer to $[18,26,27]$ for some related stability results for hybrid systems. Note that a Lyapunov candidate for a linear system of the type(3) can be selected as a piecewise quadratic function. Since (13) is an equivalent representation of the initial time-delayed system (9), the presented Theorem 4 can also be interpreted here as a stability criterion for dynamical systems with time delays (see e.g., [21]). We now can claim that under the above selection strategy for $K(\cdot)$, the resulting system (13) has a similar stable behavior as the initial NCS (9). A motivation of this fact follows from the natural interpretation of a given NCS as a hybrid system and the related theory of delayed systems (see e.g., [21]). In this paper we have studied the LQ-type problem for a class of networked and hybrid systems. It is evident that the similar problem formulation is also of a great importance for some classes of linear discrete systems of switched nature and in general for dependable control of discrete events systems.

Let us now illustrate the computational effectiveness of the proposed (quasi-optimal) LQ-based techniques and the discussed stability properties.

Example 1.

$$
\begin{aligned}
& \dot{x}(t)=x(t)+u(t), \\
& x_{0}=0 .
\end{aligned}
$$

First, we implement the computational approach proposed in Sections 34 . Note that in this case the "hybridization" of the initial delayed control system (14) contains two locations. For comparison we also study the system (14) using the analysis method proposed in [11]. The corresponding technique uses a special discrete transformation of the original networked system for a given "sampling time" $h$. The obtained dynamical behavior for $\tau=0.01$ and $h=1$ is shown in Fig.2.

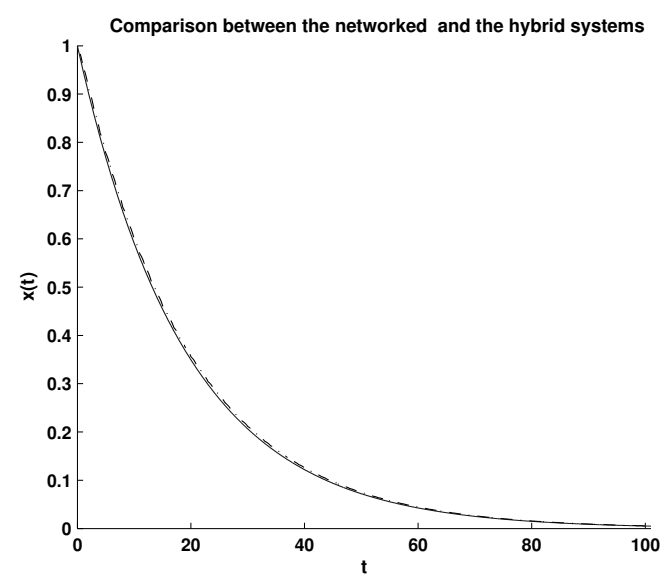

Fig. 2. Networked (the dotted line) and hybrid systems (the continuous line).

The numerical error between two above-mentioned models (the "hybridization" model and the transformed system) is presented in Fig. 3 and the resulting dynamical behavior is shown in Fig. 4. As next we present the errors between the networked and perturbed hybrid system (see Fig. 5).

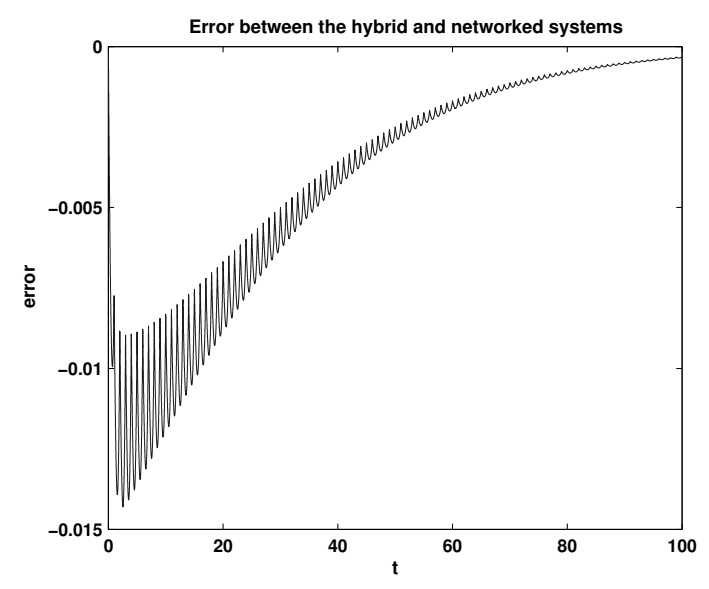

Fig. 3. Networked and hybrid systems: the error analysis.

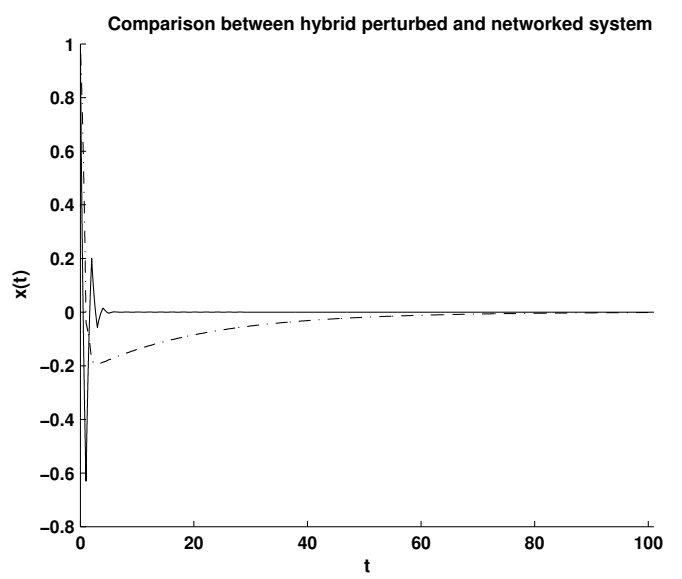

Fig. 4. Networked and hybrid control systems (dotted line networked system, continuous line hybrid system)

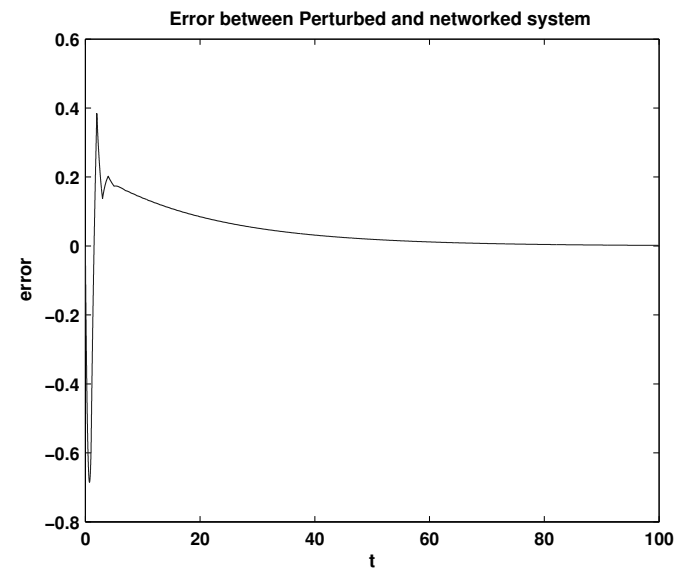

Fig. 5. Networked and perturbed hybrid system: error analysis (dotted line networked system, continuous line hybrid system)

The above computation was carried out, using the standard MATLAB packages. 


\section{CONCLUDING REMARKS}

In this paper, we have developed a new computational approach to optimal control a class of networked delayed systems. The proposed optimization approach is based on the extension of the conventional MP and DP techniques to control processes governed by linear hybrid systems. Using the switched structure of the initial delayed networked system, we study an equivalent model in terms of the hybrid dynamics. This make it possible to apply the newly developed hybrid LQ-type optimization theory to the quasi-optimal design of the NCSs under consideration. Moreover, we also deal with the stability analysis of the obtained closed-loop system with delays. The paper also deals with computational issues and contains an example of simulated control laws that stabilize the linear NCSs. We consider our numerical approach based on the above "hybridization" of the initial NCS in comparison to a conventional technique and establish stability properties of the chosen quasi-optimal feedback strategy.

Finally note, that the presented analytical and numerical techniques can also be applied to some alternative classes of linear hybrid and networked dynamical systems with delays [12]. The above LQ-based "hybridization" approach to delayed systems can also be very useful in the context of the LQ-type problems for discrete and for some classes of dependable control processes governed by discrete events systems [3].

\section{REFERENCES}

[1] K.L. Åström and B. Wittenmark, ComputerControlled Systems, Theory and Design, Prnetice Hall Inc., New Jersey, 1997.

[2] S.A. Attia, V. Azhmyakov and J. Raisch, State jump optimization for a class of hybrid autonomous systems, in: Proceedings of the 2007 IEEE MultiConference on Systems and Control, 2007, pp. 14081413.

[3] S.A. Attia, V. Azhmyakov and J. Raisch, On an optimization problem for a class of impulsive hybrid systems, Discrete Event Dynamic Systems, to appear.

[4] V. Azhmyakov, S.A. Attia, D. Gromov and J. Raisch, Necessary optimality conditions for a class of hybrid optimal control problems, Lecture Notes in Computer Science, vol. 4416, Springer, Berlin, 2007, pp. 637-640.

[5] V. Azhmyakov, S.A. Attia and J. Raisch, J., On the Maximum Principle for impulsive hybrid systems, Lecture Notes in Computer Science, vol. 4981, Springer, Berlin, 2008, pp. 30-42.

[6] V. Azhmyakov, R. Galvan-Guerra and A. Polyakov, On the Dynamic Programming method for LQ hybrid optimal control problems, Automation and Remote Control to appear in 2009.

[7] V. Azhmyakov, V. Boltyanski, A. Poznyak, Optimal control of impulsive hybrid systems, Nonlinear Analysis: Hybrid Systems, vol. 2, no. 4, 2008, pp. 1089-1097.

[8] J. Baillieul and P.J. Antsaklis, Control and communication challenges in networked real-time systems, in: Proceedings of the IEEE vol 95, no. 1, 2007, pp. 9-28.

[9] R. Bellman, Dynamic Programming, Princeton University Press, Princeton, 1957.

[10] L. Berkovitz, Optimal Control Theory, Springer, New York, 1974.
[11] M.S. Branicky, S.M. Phillips and W. Zhang, Stability of networked control systems: explicit analysis of delay, in: Proceedings of the 2000 American Control Conference, 2000, pp. 2352-2357.

[12] M.S. Branicky, Multiple Lyapunov functions and other analysis tools for switched and hybrid systems, IEEE Transactions on Automatic Control, vol. 43, no. 4, 1998, pp. 475-482.

[13] A.E. Bryson and Y.C. Ho, Applied Optimal Control, Hemisphere Publising Corp., New York, 1975.

[14] P. Caines and M.S. Shaikh, Convergence analysis of hybrid Maximum Principle optimal control algorithms, in: Proceedings of the 17th International Symposium on Mathematical Theory of Networks and Systems, 2006, pp. 2083-2088.

[15] P. Caines, M. Egerstedt, R. Malhame, A. Schoellig, A hybrid Bellman equation for bimodal systems, Lecture Notes in Computer Science, vol. 4416, Springer, Berlin, 2007, pp. 656-659.

[16] C. Cassandras, D.L. Pepyne and Y. Wardi, Optimal control of class of hybrid systems, IEEE Transactions on Automatic Control, vol. 46, 2001, pp.398-415.

[17] Z. Chen, L. Liu, L. and J. Zhang, Observer based networked control systems with network-induced time delay, in: Proceedings of the 2004 IEEE International Conference on Systems, Man and Cybernetics, 2004, pp. 3333-3337.

[18] J. Daafouz, P. Riedinger and C. Iung, Stability analysis and control synthesis for switched systems: a switched Lyapunov function approach, IEEE Transactions on Automatic Control, vol. 47, no. 11, 2002, pp. 1883-1887.

[19] M. Egerstedt, Y. Wardi and H. Axelsson, Transitiontime optimization for switched-mode dynamical systems, IEEE Transactions on Automatic Control, vol. 51, 2006, pp. 110-115.

[20] H.O. Fattorini, Infinite-Dimensional Optimization and Control Theory, Cambridge University Press, Cambridge, 1999.

[21] K. Gu, V.L. Kharitonov and J. Chen, Stability of Time-Delay Systems, Birkhäuser, New York, 2003.

[22] B. Piccoli, Hybrid systems and optimal control, in: Proceedings of the 37th IEEE Conference on Decision and Control, 1998, pp. 13-18.

[23] B. Piccoli, Necessary conditions for hybrid optimization, in: Proceedings of the 38th IEEE Conference on Decision and Control, 1999, pp. 410-415.

[24] M.S. Shaikh and P. E. Caines, On the hybrid optimal control problem: theory and algorithms, IEEE Transactions on Automatic Control, vol. 52, 2007, pp. 1587-1603.

[25] H.J. Sussmann, A maximum principle for hybrid optimization, in: Proceedings of the 38th IEEE Conference on Decision and Control, 1999, pp. 425-430.

[26] L. Xie, W. Zhao and Z. Ji, LQG control of networked control system with long time delays using deltaoperator, in: Proceedings of the 6th International Conference on Intelligent Systems Design and Applications, 2006, pp. 183-187.

[27] W. Zhang, M.S. Branicky and S.M. Phillips, Stability of networked control systems, IEEE Control Systems Magazine, vol. 21, no. 1, 2004, pp. 84-99. 